\title{
Ein organisationstheoretischer Ansatz zur Erforschung diskursiver Prozesse im Management Coaching
}

\author{
Florian Schulz ${ }^{1}$
}

Online publiziert: 10. Februar 2016

(C) Die Autor(en) 2016. Dieser Artikel ist auf Springerlink.com mit Open Access verfügbar

\begin{abstract}
Zusammenfassung Ausgehend von einer Problematisierung aktueller Entwürfe von Coaching innerhalb der interdisziplinären Organisationsforschung wird im vorliegenden Beitrag die Notwendigkeit betont den Kontext von Coaching stärker zu differenzieren, das Augenmerk auf die konstituierenden Prozesse zu richten sowie vermehrt kritische Fragen zu den Effekten der Intervention zu stellen. Ein prozessorientiertes Diskurskonzept wird vorgestellt, welches erlaubt diese Forderungen zu erfüllen sowie Coaching auf drei Prozessebenen zu betrachten: Coaching als Gespräch, als organisationale Intervention sowie als sozio-historisches Phänomen. Die konkreten Implikationen für die Forschung werden anhand von Beispielen aus einem empirischen Projekt illustriert. Damit leistet der Artikel insgesamt einen Beitrag zur Entwicklung eines alternativen theoretischen Verständnisses von Coaching, stellt methodische Möglichkeiten zur Erforschung der Prozesse dar und unterstreicht die organisationstheoretische Perspektive bei der empirischen Erforschung.
\end{abstract}

Schlüsselwörter Management Coaching · Diskursanalyse · Diskursive Prozesse · Organisationstheorie $\cdot$ Kritische Reflexion

Florian Schulz

Florian.schulz@unisg.ch

Lehrstuhl für Organisationspsychologie, Universität St. Gallen, Girtannerstrasse 6, 9010 St. Gallen, Schweiz

\section{An Organizational Theory Framework to Exploring Discursive Processes in Management Coaching}

\begin{abstract}
Based on a critical examination of current approaches to coaching within the interdisciplinary organizational studies, this contribution stresses the necessity to differentiate more strongly the context in coaching, to focus on the constitutive processes of coaching and to ask more critical questions concerning the efficacy of the interventions. A process-oriented discursive concept is presented which meets these three exigencies and allows to examine coaching along the following three processual levels: coaching as conversation, coaching as organizational intervention and coaching as socio-historical phenomenon. Concrete implications for coaching research are illustrated with the help of examples from an empirical project. As such, the article contributes to developing an alternative theoretical understanding of coaching, offers methods to investigate coaching processes and underlines the organization-theoretical perspective of the empirical approach.
\end{abstract}

Keywords Management coaching - Discourse analysis · Discursive processes - Organizational theory ·

Critical reflection

\section{Einleitung}

Der vorliegende Artikel basiert auf dem Forschungsprojekt The Psycho-Managerial Complex at Work: A Study of the Discursive Practices of Management Coaching (Schulz 2013). Dieses Projekt beschreibt und analysiert Management Coaching aus der Perspektive einer diskursorientieren 
Organisationsforschung, welche die gestaltende Funktion von Sprache bei der Konstituierung sozialer Phänomene im Arbeitskontext betont. Das Hauptargument des Beitrags ist demnach, dass sich die methodischen und theoretischen Ressourcen diskursiver Ansätze für die Erforschung des Phänomens Coaching gewinnbringend aktivieren lassen.

Ausgangspunkt des Artikels ist eine Analyse der aktuellen Literatur zum Thema Coaching in der Organisationsforschung, aus der sich die folgenden drei Möglichkeiten einer konzeptuellen Weiterentwicklung der Coachingforschung ableiten lassen. Erstens wird vorgeschlagen, Coaching als fortlaufende soziale Konstruktion zu verstehen und anhand des Kontextes zu definieren. Zweitens werden die Möglichkeiten kritisch-diskursiver Reflexion der Effekte von Coaching aufgezeigt. Drittens setzt sich der Artikel dafür ein, die in und durch Management Coaching ablaufenden Prozesse auf drei zusammenhängenden Ebenen zu beschrieben: die Dynamik des Gespräches zwischen Coach und Coachee, die Einbettung der Coaching-Intervention im Arbeitsumfeld sowie die Wechselwirkung zwischen der Veränderung der Arbeitswelt und Coaching als sozio-historischem Phänomen. Abgeleitet von einer prozessorientierten Diskurstheorie wird schließlich anhand von konkreten empirischen Bespielen aufgezeigt wie sich die drei vorgeschlagenen Weiterentwicklungen methodisch umsetzen lassen. Das übergeordnete Anliegen des Beitrags ist damit aufzuzeigen, wie sich anhand eines kritisch distanzierten Forschungsansatzes die verschiedenen mit Coaching assoziierten Prozesse analysieren lassen und dadurch ein alternativer Beitrag zur wissenschaftlichen Auseinandersetzung mit dem sich rasant entwickelnden Phänomen machen lässt.

\section{Coaching aus der Perspektive der Organisationsforschung}

Mit starken Bezügen zur allgemeinen Organisationssoziologie und -psychologie sowie der Managementforschung gilt das Hauptinteresse der interdisziplinär ausgerichteten Organisationsforschung (im Folgenden OF abgekürzt) dem Verstehen von Phänomenen innerhalb der Sphäre der Arbeitswelt (Clegg et al. 2006). Dabei gilt es einerseits die Entstehung, Aufrechterhaltung und Veränderung von Organisationen und deren Beziehung zur Außenwelt zu begreifen. Andererseits, und damit unweigerlich verbunden, ist die Frage danach wie sich Menschen organisieren und damit die Voraussetzung für zielgerichtete Handlungen und letztlich einer Wertschöpfung schaffen (Hernes und Maitlis 2012). Typische Themen der OF sind z. B. wie Mitarbeitende kommunizieren, welchen Einfluss spezifische Praktiken auf die Produktivität der Organisation haben, wie politische Kräfte offen oder verdeckt Machtkämpfe in
Unternehmen austragen oder wie sich eine Unternehmenskultur über die Zeit hinweg entwickelt (Deetz 2003). Aufgrund der Integration verschiedener Wissenschaftstraditionen innerhalb der OF werden sowohl quantitative Studien mit funktionalistischem Forschungsparadigma als auch qualitative Studien, welche in ihrer Theoriebildung an den Sozial- und Geisteswissenschaftlichen angelehnt sind, in den führenden Zeitschriften der OF publiziert und diskutiert.

Um die Bedeutung von Coaching in der aktuellen Literatur der OF kommentieren zu können, wurde eine systematische Literaturrecherche (Schulz 2013) durchgeführt. Es soll an dieser Stelle deutlich darauf hingewiesen werden, dass diese Literaturrecherche nicht den Anspruch erhebt/erhob umfassend zu sein. Die zitierte Recherche war auf die OF bezogen und basierte auf den wichtigsten 61 Zeitschriften der OF, also englischsprachige Journals mit ImpactFactor aus den Bereichen der Managementforschung, der Organisationspsychologie sowie Organisationssoziologie. Betrachtet man die Diskussionen zum Thema Coaching in diesem Literaturkorpus lässt sich zunächst sagen, dass Coaching zwar noch kein etablierter Forschungsgegenstand der OF ist, es sich jedoch eine zunehmende Zahl von Artikeln zum Thema finden lassen. Bei genauerem Hinsehen ergibt sich das Bild, dass sich dieser Forschungsstrang theoretisch wie methodisch eher noch in einem frühen Stadium der Ausdifferenzierung befindet. Auch ist der von Feldman (2005) geforderte Blick in die Black Box Coaching, also die Frage nach den tatsächlich ablaufenden Prozessen und angewendeten Praktiken der Intervention, noch kaum getätigt worden.

An dieser Stelle wird die Frage aufgeworfen, wie sich die bestehende Forschungslandschaft weiter entwickeln lässt. Anstelle einer Suche nach Forschungslücken innerhalb der bestehenden Beschreibungen von Coaching wird vorgeschlagen eine Problematisierung der bestehenden Diskussionen vorzunehmen (Alvesson und Sandberg 2011). Ziel einer solchen Problematisierung ist es wenig repräsentierte und neue Perspektiven auf ein Phänomen in der OF aufzuzeigen und damit grundlegende Entwicklungsräume aufzuzeigen. In diesem Sinne ergeben sich drei Möglichkeiten, welche eine Forschung, mit dem Interesse die konkreten Praktiken und Prozesse von Coaching zu beschreiben, zu analysieren und zu diskutieren, voran bringt:

Erstens erscheinen aktuelle Beiträge defragmentiert und es mangelt an einer genauen Differenzierung des Forschungsgegenstandes „Coaching“. Die gefundenen Artikel in der Literaturrecherche nehmen untereinander wenig Bezug, wodurch das Feld sich konzeptuell inkohärent gibt. Ein Hauptgrund dafür scheint, dass das, was als „Coaching“ bezeichnet wird, bei genauerem Hinsehen sehr verschiedene soziale Interaktionen sind. Mit Coaching werden unterschiedliche Formen der personenzentrierten 
Personalentwicklung, Mitarbeitergespräche im Rahmen der Personalführung, eine Unterstützung von Verkaufs- und Akquisetrainings, Gruppendynamische Seminare sowie nicht näher definierte Beratungsformate zusammengefasst. Gemeinsam ist allen Definitionen von Coaching, dass diese normative Aussagen über die Funktion und den Charakter von Coaching machen. Defragmentierung der Forschungsdiskussionen entsteht dann aus dem Umstand, dass sich diese normativen Beschreibungen z. T. deutlich voneinander unterscheiden. Daraus entsteht der Eindruck, dass eventuell mehr gewonnen wäre, wenn die Forschung auf diese Definitionen von Coaching verzichten würde und sich stattdessen stärker der Ebene der Prozesse und Praktiken der Intervention zuwendet. In jedem Fall scheint eine genaue Beschreibung und Eingrenzung des jeweiligen Forschungsfeldes die essentielle Voraussetzung für den Aufbau von kohärenten Forschungsgemeinschaften zu sein.

Zweitens bauen die Autor/innen ihre Konzeptualisierungen von Coaching zumeist auf den psychodynamischen, lerntheoretischen, narrativ-diskursiven, humanistischen und konstruktivistisch-systemischen Veränderungsansätzen der Psychotherapie auf (z. B. Ducharme 2004; Gray 2006; Sherin und Caiger 2004). Zeitgleich versuchen Autor/innen sich aber auch möglichst weit von der Psychotherapie sowie anderen Beratungsformaten zu distanzieren, auch indem der Ursprung der Veränderungstheorien nicht explizit benannt wird (Price 2009). An diesem Umstand anknüpfend wird Coaching von Clegg et al. (2007) eine „ontologische Unsicherheit“ attestiert, also ein Unvermögen, die eigene Identität ohne starke Brüche zum Ursprung und ohne Widersprüche $\mathrm{zu}$ formulieren. Ein Ausweg aus diesem Umstand könnte der Einsatz alternativer und eigenständiger theoretischer Modelle sein, eine stärkere Akzentuierung der Rolle des Kontextes sowie die bereits herausgestellte genaue Beschreibung und Analyse der im Coaching ablaufenden Dynamiken.

Drittens stellt man fest, dass die publizierten Artikel fast durchgängig eine affirmative Haltung zum Forschungsgegenstand Coaching einnehmen. Dies wird durch den Umstand unterstrichen, dass aus den biographischen Angaben der Autor/innen häufig erkennbar wird, dass sie selbst Anbieter von Coaching-Dienstleistungen sind. Möglicherweise kann eine Vielzahl der aktuellen Publikationen auf dem Feld der OF als Versuch gewertet werden, Coaching als Dienstleistung mittels des hohen Renommees akademischer Institutionen weiter zu legitimieren. Während das Engagement der Autor/innen wissenschaftliche Belege für die positive Wirksamkeit von Coaching zu finden grundsätzlich wenig bedenklich erscheint, ergibt sich daraus eine mögliche Problematik für die weitere Entwicklung des Feldes, da es zum Teil noch an kritischer Distanz zum Forschungsgegenstand fehlt. Eine Möglichkeit diesem Umstand beizukommen ist das Stellen kritischer Fragen
(Alvesson und Willmott 1992), welche die Reflexion, und damit die Fortentwicklung des Feldes, befördern können.

Aus der Analyse der OF-Literatur zur Thema Coaching ergeben sich also drei mögliche Handlungsempfehlungen zur weiteren Entwicklung der Forschung (vgl. Schulz 2013): die genaue Beschreibung des Forschungsgegenstandes, die Entwicklung alternativer Rahmenkonzepte sowie die Entwicklung einer kritischen Position zum Forschungsgegenstand durch kritisch-informierte Forschungsfragen.

\section{Möglichkeiten der diskursiven Organisationsforschung zur Erforschung von Management Coaching}

Als Lösungsvorschlag für die soeben aufgezeigten Problematisierungen wird das Forschungsparadigma einer diskursorientierten Prozessforschung vorgestellt. Im ersten Teil dieses Abschnittes werden zunächst grundlegende Annahmen der diskursiven OF besprochen, bevor im zweiten Abschnitt eine Übersetzung auf das Phänomen Coaching vorgenommen wird. Dies ist letztlich eine Vorbereitung für den dann folgenden Abschnitt, in dem konkrete Beispiele für die methodische Ausgestaltung empirischer Analysen gegeben werden.

\subsection{Die diskursive Organisationsforschung}

Die diskursorientierte $\mathrm{OF}$ ist ein interdisziplinärer Forschungsansatz in der unter anderem Strömungen der Foucaultschen Diskursanalyse, der Diskurspsychologie sowie aus der linguistischen Analyse von Texten zusammenfließen. Diskursive Ansätze haben sich seit den 1990er Jahren in der Organisationsforschung fest etabliert, da sie sowohl ein theoretisches als auch ein methodologisches Rahmenkonzept zur Erforschung sozialer Interaktionen und der Produktion von Wissen liefern (Phillips und Di Domenico 2009) und werden ,zunehmend als das wichtigste, empirisch zugängliche, Phänomen der Sozial- und Organisationsforschung anerkannt" (Alvesson und Kärreman 2011, S. 1137'). Als Ausdruck des sozial konstruktionistischen Paradigmas wird die Bedeutung und organisierende Funktion der Sprache bei der Konstituierung sozialer Prozesse betont (Grant et al. 2004). Auch wird grundsätzlich angenommen, dass Wissen durch eine soziale Interaktion, in der die Akteure allgemeine Wahrheiten konstruieren und dessen Bedeutung konsolidieren oder herausfordern, erzeugt wird (Lawless et al. 2011). Damit lässt sich das allgemeine Anliegen der OF präzisieren: Die diskursorientierte OF interessiert sich dafür wie Organisationen sich durch Sprache konstituieren und, damit

${ }^{1}$ Übersetzung aus dem Englischen durch den Autor. 
verbunden, wie Menschen sich und andere durch diskursive Prozesse organisieren.

Darüber hinaus kann man innerhalb der diskursorientierten OF nach Alvesson und Kärreman (2011) zwischen zwei grundsätzlichen Ansätzen unterscheiden. Text-fokussierte Studien und Paradigmen-orientierte Diskursanalysen bilden zwei Pole der Forschungslandschaft, wobei sich erstere eher als Mikroforschung und letztere eher als Makroforschung verstehen lassen. Text-fokussierte Studien, welche auch als mikro-diskursive Studien, Gesprächsanalysen oder text-basierte Analysen bezeichnet werden, richten das Augenmerk auf die Konstruktion der sozialen Welt durch sprachliche Interaktionen. Im Zentrum des Interesses steht die linguistische Analyse von Texten, also alle Formen gesprochener und geschriebener Sprache, welche als soziale Praktiken verstanden werden (Wood und Kroger 2000). Text-fokussierte Studien setzen Diskurs häufig mit lokal produzierten, sprachlichen Handlungen gleich und gehen davon aus, dass diese über den Kontext ihrer Entstehung hinaus nur wenig verallgemeinerbare Gültigkeit besitzen (Alvesson und Kärreman 2011). Studien, die ein solches Verständnis von Diskurs haben, untersuchen meist in induktiver Form die Ausprägung und Wirkung die Texte z. B. in sozialen Interaktionen, den Medien, wichtigen Dokumenten etc. hervorrufen. Demgegenüber stehen Paradigmen-orientierte Diskursanalysen, welche auch als Foucault-basierte Studien, Makro-Diskursive Studien oder Studien zu Ideologie-basierten Weltsichten bezeichnet werden (Townley 1993; Barratt 2003). Die Position betont, dass soziale Realität durch historisch geformte Ideen geschaffen und reproduziert wird. Entsprechend beschäftigt sich diese Form der Forschung weniger mit der Analyse alltäglicher Sprachaktivitäten und mehr mit der Suche nach wiederkehrenden Mustern von Ideen und deren Bezug zum sozio-historischen Kontext. Zentraler Ausgangspunkt für dieses Forschungsparadigma sind die Werke Foucaults, in denen er sich insbesondere mit der gesellschaftlich-historischen Entwicklung von Wissen, Macht und Mechanismen der Kontrolle kritisch auseinandersetzt. Es wird dabei angenommen, dass die in einer Zeit vorherrschenden Diskurse, also die wiederkehrenden Systeme von Ideen, eine relative Konstanz und Stabilität aufweisen und über eine Reihe von Situationen ihre Wirkung entfalten.

\subsection{Aktivierung theoretischer Ressourcen der diskursiven Organisationsforschung zur Erforschung von Management Coaching}

Die im obigen Abschnitt aufgezeigten Annahmen und die zwei Richtungen diskursorientierter OF sollen nun dabei behilflich sein die Forschung zum Thema Coaching weiter zu entwickeln. Zunächst zum Aspekt einer genauen Klärung des Forschungsfeldes „Coaching“. Über- trägt man die Grundannahmen der oben referierten diskursiver Ansätze auf Coaching, so muss man Coaching als soziale Konstruktion verstehen. Damit werden normative Beschreibungen der Funktion und Sinnhaftigkeit von Coaching nicht der Ausgangspunkt der Forschung, sondern der Gegenstand derselben. Es wird angenommen, dass wir mehr über die Wirkung des Phänomens erfahren können, wenn wir die multiplen Bedeutungen für Organisationen wie für Individuen begreifen und der Frage nachgehen, wie diese Bedeutungen positioniert, angefochten oder stillschweigend übergangen werden. Aus der Perspektive einer diskursorientierten OF wird also danach gefragt, wie Coaching mit und durch Sprache abgebildet wird und Wirkung entfaltet. Entsprechend wird vorgeschlagen als Ausgangspunkt nicht Coaching zu definieren, sondern die organisationalen Rahmenbedingungen innerhalb derer diese spezifische Form sozialer Interaktionen stattfindet. In diesem Sinne wurde in dem hier referierten Forschungsprojekt (Schulz 2013) der untersuchte Forschungsgegenstand „Management Coaching“ wie folgt definiert: „Management Coaching ist ein von einer Organisation bezahltes Gespräch, das als ,Coaching' bezeichnet wird. Dieses Gespräch findet zwischen einem Angestellten der Organisation und einer von der Organisation beauftragten Person, die sich als ,Coach" betitelt, statt" (Schulz 2013, S. 44). Diese Definition von Management Coaching ermöglicht dem Phänomen „Coaching“ ohne normativ geladene Vornahme zu begegnen. Anders ausgedrückt sind die normativen Definitionen von Coaching nicht Ausgangspunkt sondern Untersuchungsgegenstand dieser Forschung. Damit ist auch die Forderung nach mehr Distanz zum Forschungsobjekt zu Teilen erfüllbar. Des Weiteren macht die Definition auch deutlich, dass es sich hier um ein Phänomen handelt, das fest in der Arbeitswelt verankert ist. Implizit schließt diese Definition damit auch an bestehende Diskussionen $\mathrm{zu}$ Personalentwicklungs-Interventionen innerhalb des Human Ressource Management an, wodurch die Diskussionen um das Phänomen Coaching eine Öffnung hin zu bestehenden Auseinandersetzungen bekommt. Letztlich ist diese Definition auch eine Voraussetzung für eine genaue Analyse der diskursiven Prozesse, die innerhalb dieses Rahmens stattfinden.

Die diskursiven Ansätze der OF greifen auf eine lange Tradition des kritischen Denkens u. a. des marxistischen Materialismus, der Frankfurter Schule, feministischer und post-kolonialer Studien und der Queer Theorie zurück (Alvesson und Willmott 1992). Häufig zitierte Werke sind dabei die von Michel Foucault, Pierre Bourdieu, Jacques Derrida, Gilles Deleuze und Judith Butler (van Dijk 2001). Die kritische Diskursanalyse ermöglicht Einsichten in die Art und Weise wie diskursive Strukturen das Beziehungsgefüge zwischen Macht und Dominanz in einer Gesellschaft inszenieren, bestätigen, legitimieren, reproduzieren 
Tab. 1 Übersetzung auf Forschungsmethoden und -designs

\begin{tabular}{|c|c|c|c|c|}
\hline Ebene von Coaching & Forschungsperspektive & Prozessorientierung & Kritischer Reflexion & Empirisches Material \\
\hline Gespräch & $\begin{array}{l}\text { Wie wird das Ge- } \\
\text { spräch wechselseitig } \\
\text { inszeniert? }\end{array}$ & $\begin{array}{l}\text { Wie positionieren sich die } \\
\text { Akteure zueinander und über } \\
\text { den Verlauf der Zeit hinweg? }\end{array}$ & $\begin{array}{l}\text { Anwendung rhetorische Mittel } \\
\text { und Autorität damit Coachee } \\
\text { Narration übernimmt }\end{array}$ & $\begin{array}{l}\text { Transkriptionen und ethno- } \\
\text { graphische Aufzeichnungen } \\
\text { von Gesprächen }\end{array}$ \\
\hline $\begin{array}{l}\text { Organisationale } \\
\text { Intervention }\end{array}$ & $\begin{array}{l}\text { Wie wird MC } \\
\text { legitimiert? }\end{array}$ & $\begin{array}{l}\text { In welcher Relation stehen } \\
\text { unterschiedliche Diskurse } \\
\text { zueinander? }\end{array}$ & $\begin{array}{l}\text { Einfluss verdeckter Ziele } \\
\text { und unternehmenspolitischer } \\
\text { Dynamik }\end{array}$ & $\begin{array}{l}\text { Interviews, Interne } \\
\text { Dokumente und ethno- } \\
\text { graphische Notizen }\end{array}$ \\
\hline $\begin{array}{l}\text { Sozio-historisches } \\
\text { Phänomen }\end{array}$ & $\begin{array}{l}\text { Wie trägt MC zur Ver- } \\
\text { änderung der Arbeits- } \\
\text { welt bei? }\end{array}$ & $\begin{array}{l}\text { Wie etablieren sich neue } \\
\text { Arbeitspraktiken? }\end{array}$ & $\begin{array}{l}\text { Steigerung der Intensität der } \\
\text { emotionalen Arbeit, der Selbst- } \\
\text { ausbeutung im Spätkapitalismus }\end{array}$ & $\begin{array}{l}\text { Medienberichte, Praxis- } \\
\text { literatur, Internetseiten } \\
\text { und Broschüren von } \\
\text { Ausbildungsinstituten }\end{array}$ \\
\hline
\end{tabular}

oder herausfordern (van Dijk 2001, S. 353). Darüber hinaus kann kritisches Denken zu einer emanzipatorischen Position gegenüber dominierenden Diskursen, durch deren Dekonstruktion, führen (Alvesson und Willmott 1992). Bezogen auf Coaching bedeutet dies, dass nicht nur Fragen bezüglich des Auftretens möglicher Nebenwirkungen, im Sinne des medizinischen Modells von Veränderung, gestellt werden (De Haan et al. 2010), sondern eben auch kritische Frage durch die oben genannten theoretischen Konzepte informiert sind. Das meint, dass man der Frage nachgeht, wie Coaching Gegenstand politischer Dynamiken wird und die dominierenden Machtstrukturen in einem Unternehmen verstärkt oder z. B. heterosexuelle und maskuline Normen reproduziert.

Die Beschreibung von Coaching als soziale Konstruktion, sowie die Annäherung an kritisch informierte Fragestellungen soll nun weiter ergänzt werden durch eine zusammenfassende Forschungsperspektive. Konkret wird vorgeschlagen, angelehnt an das integrative Diskursmodel von Fairclough (1992; siehe auch Nielsen und Nørreklit 2009), Coaching auf drei, miteinander zusammenhängenden, Ebenen diskursiver Prozesse zu unterscheiden. Diese sind Coaching als Gespräch, Coaching als organisationale Intervention sowie Coaching als sozio-historisches Phänomen. Durch die Betonung, dass es sich auf den drei Ebenen um diskursive Prozesse handelt, soll der fortlaufende Charakter von Coaching als Diskurs unterstrichen werden. Damit, so der Vorschlag, lässt sich auch die den im Abschn. 3.1 vorgestellten diskursiven Richtungen der OF implizite Makro-Mikro Dichotomie überwinden. Ich folge damit der Aussage von Cooren und Kollegen, dass die paradigmatischen Makro-Diskurse kontinuierlich in Form von Texten reproduziert, aufrechterhalten und von einem Punkt zum anderen übertragen werden müssen, da sie sonst in der Bedeutungslosigkeit versinken würden und keine Wirkung in der Welt hätten (Cooren et al. 2007).

\section{4 Übersetzung auf Forschungsmethoden und -designs}

An dieser Stelle sollen konkrete Implikationen für die Forschung aufgezeigt werden, unter Bezug auf die ausführlichen Analysen meines Promotionsprojektes (Schulz 2013). Tabelle 1 fasst dabei die Fragestellungen, das verwendete empirische Material, die angewendeten theoretischen Konzepte sowie die kritische Fragen zusammen.

\subsection{Coaching als Gespräch}

Analysiert man Management Coaching auf der Ebene des Gespräches, werden die sprachlichen Aktivitäten, die zwischen Coach und Manager/in ablaufen, fokussiert. Dabei wird betont, dass Sprache auch immer die Qualitäten einer Handlung besitzt, deren Dynamik und Wirkung untersucht werden kann. Die Voraussetzung für eine solche Analyse sind entweder ethnographische Protokolle der Gespräche oder Transkriptionen solcher Gespräche. Dabei haben Transkriptionen eine deutlich höhere Informationsdichte und geben das Gespräch genauer wieder, sind aber aufgrund von Problemen des Zugangs auch deutlich schwieriger zu bekommen. Aus Perspektive der OF lassen sich eine Reihe verschiedener Fragestellungen anhand eines diskursiven Vorgehens verfolgen (Graf 2012). Interessant ist dabei insbesondere wie aus der Dynamik des Gespräches Rückschlüsse auf die organisationalen Intentionen und Effekte von Management Coaching gezogen werden können. Als Beispiel einer empirischen Forschung sei hier auf die Studie Talk at work: An analysis of the discursive processes of management coaching conversations (Schulz und Steyaert 2014) verwiesen. Ausgehend von einem diskursiven Prozessmodell untersucht die Studie einerseits die Interaktionen zwischen Coach und Manager/in, also was Coach und Manager/in auf der Ebene des Gespräches „machen“. Zweitens stellt die Studie die temporalen Veränderungen verschiedener Interpretationsmuster für die Situation des Managers heraus und greift dabei auf narrativ-diskursive Veränderungstheorien zurück. Basierend auf der Analyse 
dreier authentischer Gespräche argumentiert die Studie, dass der Coach wiederkehrende rhetorische/diskursive Praktiken verwendet damit der Manager seine Deutungen annimmt. Dabei zeigt die Studie auf, wie es dem Coach anhand einer Strategie, bezeichnet als „emphatische Überzeugung“, gelingt, dass der Manager die bevorzugten Deutungen des Coachs annimmt und sich zu Eigen macht. Letztlich bringt dieser Beitrag die Diskussion auf die Art und Weise, wie solche Management Coaching Gespräche als personenzentrierte HR Maßnahme verstanden werden können, die im Sinne des Unternehmens das unternehmerische Selbst der Person fördern und damit dessen Produktivität dienen. Dieser Aspekt der Diskussion weist auch schon auf die nachfolgende Ebene von Coaching hin.

\subsection{Coaching als organisationale Intervention}

Die zweite Ebene - MC als organisationale Intervention bildet die Gespräche über das Coaching Gespräch ab und kann auch als Meso-Ebene betrachtet werden. Hier stellt sich also die Frage nach der sozialen Konstruktion der Intervention und damit wie der Verwendungszweck und die Legitimität in spezifischen Unternehmen offiziell, aber auch hinter verschlossenen Türen, ausgehandelt werden. $\mathrm{Da}$ es zumeist eine Vielzahl miteinander konkurrierender Antworten auf diese Fragen gibt, lässt sich darüber hinaus betrachten, wie die verschiedenen diskursiven Konstruktionen zueinander in Beziehung stehen und welche Spannungen, Brüche oder Fusionen dies hervorruft. Bedeutend ist wie $\mathrm{MC}$ von den beteiligten Akteuren, also v. a. Coaches, HR-Managern, Vorgesetzten, CEOs und den betroffenen Coachees diskursiv konstruiert wird, besonders im Hinblick darauf, wie sie den Einsatz der aufgebrachten Ressourcen (Zeit, Geld, Aufmerksamkeit) legitimieren. Entsprechend bestehen empirische Daten aus Interviews, der ethnographischen Teilnahme am Arbeitsalltag, Emails oder Dokumenten, die Aufschluss darüber geben, wie die Akteure über Management Coaching sprechen.

Beispielsweise konnte eine Studie von Schulz (2013) aufzeigen, wie sowohl psychotherapeutische als auch Management Diskurse zur Legitimierung von Management Coaching herangezogen werden. Weiterhin weist die Studie darauf hin, wie diese Diskurse zueinander in Beziehung gebracht werden, in dem vor allem der Managementdiskurs die psychotherapeutischen Gesichtspunkte assimiliert; damit werden in der Legitimierung von Coaching die marktwirtschaftlichen Gesichtspunkte in den Vordergrund gerückt. Eine solche Perspektive ermöglicht es somit also, die Beziehung zwischen psychotherapeutischen und Management-Diskursen zur Legitimierung von Coaching zu analysieren und aufzuzeigen, wie die Logik des Managements in die psychotherapeutischen Ansätze zum Zwecke der Wertsteigerung für das Unternehmen assimiliert wurde.

\subsection{Coaching als sozio-historisches Phänomen}

Die dritte Perspektive versucht $\mathrm{zu}$ verstehen, welche Bedeutung Management Coaching als sozio-historisches Phänomen einnimmt. Damit werden die wechselseitigen Bezüge zwischen der Entwicklung von Coaching und den Veränderungen der dominierenden Praktiken der Arbeitswelt aufgezeigt. Es stellt sich hier insbesondere die Frage, wieso Coaching gerade in dieser Zeit und in dieser Kultur als Dienstleistung so erfolgreich werden konnte und welche längerfristigen Konsequenzen seine Etablierung wiederum auf die Organisation der Arbeitswelt hat. Eine solche Analyse zeigt damit die größeren Muster gesellschaftlicher Zusammenhänge auf und bedient sich, um dies zu bewerkstelligen, zumeist eines Foucaultschen Verständnisses von Diskurs, welches die paradigmatische Dynamik auf einer Makro-Ebene diskutiert. Als empirische Materialien eignen sich Dokumente, die einflussreiche Ideen und Wertvorstellungen der Zeit widerspiegeln, also z. B. CoachingBeststeller, Medienberichte über Coaching, Internetseiten von Coachingverbänden und Ausbildungsinstituten, häufig verwendete Definitionen von Coaching oder Richtlinien zum Einsatz von Coaching von Unternehmen. Als Beispiel sei die Diskussion des Zusammenhangs zwischen Coaching und dem vielfach festgestellten Trend hin zu einer Emotionalisierung (Thrift 2005) sowie Psychologisierung (Illouz 2008) der Arbeitswelt genannt (Schulz 2013). Hinweis dafür ist die eingangs erwähnte Verwendung psychotherapeutischer Theorien und Praktiken im Coaching, welche besonders in der Lektüre von gängigen „How-To“ Büchern sehr zu Tage treten. Entsprechend kann argumentiert werden, dass es durch die Verwendung des Begriffs „Coaching“, welcher an die Metapher des Hochleistungssportes anknüpft, möglich war Psycho-Praktiken in die Arbeitswelt zu transportieren. Neben aller positiven Potentiale für die Arbeitnehmer befördert diese Entwicklung auch den Umstand, dass Arbeitnehmer heutzutage fortwährend an ihren positiven Emotionen und Motivation arbeiten sollen umso dem Unternehmen ein möglichst hohes Engagement entgegen bringen zu können. Letztlich, so eine Gegenposition zur affirmativen Betrachtung von Coaching, kann es unter Umständen auch die Selbstausbeutung von Arbeitnehmern fördern.

Zusammenfassend lässt sich sagen, dass es der in diesem Beitrag vorgestellte diskursive Ansatz ermöglicht hat Coaching als soziale Konstruktion zu verstehen. Dies erlaubt es die sprachlichen Prozesse, durch die Coaching konstituiert wird, zu differenzieren. Bedeutung hat nicht nur das Gespräch zwischen Coach und Coachee, sondern insbesondere auch wie dieses Gespräch gerahmt wird und welche gesellschaftlichen Schablonen es bedient und fördert. Dabei ergänzen sich die drei Perspektiven und sind untrennbar miteinander verwoben. Mit den vorgestellten 
empirischen Beispielen wird unterstrichen, dass es dieses Konzept in der Tat ermöglicht die „black box Coaching“ zu öffnen und dadurch, anhand von konkreten Daten, die Intervention kritische zu reflektieren.

Open Access Dieser Artikel wird unter der Creative Commons Namensnennung 4.0 International Lizenz (http://creativecommons. org/licenses/by/4.0/deed.de) veröffentlicht, welche die uneingeschränkte Nutzung, Verbreitung und Wiedergabe für beliebige Zwecke erlaubt, sofern Sie den/die ursprünglichen Autor(en) und die Quelle ordnungsgemäß nennen, einen Link zur Creative Commons Lizenz beifügen und angeben, ob Änderungen vorgenommen wurden.

\section{Literatur}

Alvesson, M., \& Kärreman, D. (2011). Decolonializing discourse: Critical reflections on organizational discourse analysis. Human Relations, 64(9), 1121-1146.

Alvesson, M., \& Sandberg, J. (2011). Generating research questions through problematization. Academy of Management Review, $36(2), 247-271$.

Alvesson, M., \& Willmott, H. (1992). Critical management studies. London: Sage.

Barratt, E. (2003). Foucault, HRM and the Ethos of the critical management scholar. Journal of Management Studies, 40(5), 1069-1087.

Clegg, S. R., Nord, W., \& Hardy, C. (2006). The Sage handbook of organization studies (2. Aufl.). London: Sage.

Clegg, S. R., Rhodes, C., \& Kornberger, M. (2007). Desperately seeking legitimacy: Organizational identity and emerging industries. Organization Studies, 28(4), 495.

Cooren, F., Matte, F., Taylor, J. R., \& Vasquez, C. (2007). A humanitarian organization in action: Organizational discourse as an immutable mobile. Discourse \& Communication, 1(2), 153-190.

De Haan, E., Bertie, C., Day, A., \& Sills, C. (2010). Clients' critical moments of coaching: Toward a "client model“ of executive coaching. Academy of Management Learning \& Education, 9(4), 607-621.

Deetz, S. (2003). Disceplinary power, conflict supression and human resource management. In M. Alvesson \& H. Willmott (Hrsg.), Studying management critically (S. 23-45). London: Sage.

Dijk, T. A. V. (2001). Critical Discourse Analysis. In D. Tannen, D. Schiffrin \& H. Hamilton (Hrsg.), Handbook of discourse analysis (S. 352-371). Oxford: Blackwell.

Ducharme, M. J. (2004). The cognitive-behavioral approach to executive coaching. Consulting Psychology Journal: Practice \& Research, 56(4), 214-224.
Fairclough, N. (1992). Discourse and text: Linguistic and intertextual analysis within discourse analysis. Discourse \& Society, 3(2), 193-217.

Feldman, D. C. (2005). Executive coaching: A review and agenda for future research. Journal of Management, 31(6), 829-848.

Graf, E. (2012). , Then I will tell you maybe a little bit about the procedure - Constructing professional identities where there is not yet a profession: The case of executive coaching. In J. Angouri \& M. Marra (Hrsg.), Constructing identities at work (S. 129-151). Basingstoke: Palgrave Macmillan.

Grant, D., Hardy, C., Oswick, C., \& Putnam, L. (2004). The Sage handbook of organizational discourse. London: Sage Publications.

Gray, D. E. (2006). Executive coaching: Towards a dynamic alliance of psychotherapy and transformative learning processes. Management Learning, 37(4), 475.

Hernes, T., \& Maitlis, S. (2012). Process, sensemaking, and organizing (Auflage: Reprint). Oxford: Oxford University Press.

Illouz, E. (2008). Saving the modern soul: therapy, emotions, and the culture of self-help. Berkeley, CA: University of California Press.

Lawless, A., Sambrook, S., Garavan, T., \& Valentin, C. (2011). A discourse approach to theorising HRD: Opening a discursive space. Journal of European Industrial Training, 35(3), 264-275.

Nielsen, A. E., \& Nørreklit, H. (2009). A discourse analysis of the disciplinary power of management coaching. Society and Business Review, 4(3), 202-214.

Phillips, N., \& Di Domenico, M. (2009). Discourse analysis in organizational research: Methods and debates. In D. Buchanan \& A. Bryman (Hrsg.), The SAGE handbook of organizational research methods (S. 549-565). London: Sage.

Price, J. (2009). The coaching/therapy boundary in organizational coaching. Coaching: An International Journal of Theory, Research and Practice, 2(2), 135.

Schulz, F. (2013). The Psycho-Managerial complex at work: A study of the discursive practices of management coaching. St. Gallen: University of St. Gallen.

Schulz, F., \& Steyaert, C. (2014). Studying talk-at-work. An analysis of the discursive processes of management coaching conversations. In F. Cooren, E. Vaara, A. Langley, \& H. Tsoukas (Hrsg.), Language and communication at work: Discourse, Narrativity and Organizing (vol. 4) (S. 173-196). Oxford: Oxford University Press.

Sherin, J., \& Caiger, L. (2004). Rational-emotive behavior therapy: A behavioral change model for executive coaching? Consulting Psychology Journal: Practice \& Research, 56(4), 225-233.

Thrift, N. (2005). Knowing Capitalism. London: Sage.

Townley, B. (1993). Foucault, power/knowledge, and its relevance for human resource management. Academy of Management Review, $18(3), 518-545$.

Wood, L. A., \& Kroger, R. O. (2000). Doing discourse analysis: Methods for studying action in talk and text. London: Sage. 